\title{
POLÍTICAS INSTITUCIONALES PARA FAVORECER EL ACCESO Y LA PERMANENCIA EN UNIVERSIDADES ARGENTINAS: UN ANÁLISIS DE CUATRO INSTITUCIONES DEL CONURBANO BONAERENSE
}

\author{
Institutional policies to promote access and permanence in Argentinian \\ universities: An analysis of four institutions from the Great Buenos Aires area
}

http://dx.doi.org/10.22235/pe.v10i1.1363

JORGE M. GOROSTIAGA*1

KARINA F. LASTRA ${ }^{* * 2}$

STELLA MARIS MUIÑOS DE BRITOS**3

${ }^{*}$ Consejo Nacional de Investigaciones Científicas y Técnicas. Argentina

**Universidad Nacional de San Martín. Argentina

Correspondencia: Jorge M. Gorostiaga, Consejo Nacional

Recibido: 18-05- 2016

de Investigaciones Científicas y Técnicas. Argentina.

Revisado: 29-07-2016

Correo electrónico: jorgegoros@gmail.com

Aceptado: 30-09-2016

\footnotetext{
${ }^{1}$ Licenciado en Ciencia Política, Universidad del Salvador, Argentina. Ph.D. Social and Comparative Analysis in Education, University of Pittsburgh, Estados Unidos. Investigador adjunto del Consejo Nacional de Investigaciones Científicas y Técnicas, Argentina. Profesor titular en la Universidad Nacional de San Martín (UNSAM), Argentina. Profesor de posgrado en la Universidad Torcuato Di Tella, Argentina.

2 Profesora en Ciencias de la Educación, Universidad Nacional de La Plata, Argentina. Doctoranda con tema de tesis: "Investigación educativa en Argentina: perspectivas, epistemológicas, históricas y políticas", Universidad Nacional de Quilmes, Argentina. Profesora adjunta regular de la Cátedra Pedagogía I, Universidad Nacional de San Martín, Argentina. Investigadora en el Centro de Estudios Interdisciplinarios en Educación, Cultura y Sociedad, Universidad Nacional de San Martín, Argentina.

${ }^{3}$ Licenciada y profesora de Historia del Arte, Facultad de Filosofía y Letras, Universidad de Buenos Aires, Argentina. Especialista en Ciencias Sociales con mención en Gestión Educativa, Facultad Latinoamericana de Ciencias Sociales, sede Argentina. Magíster en Gestión Educativa, Universidad Nacional de San Martín, Argentina. Doctorado en Educación (tesis en curso), Universidad Nacional de Tres de Febrero, Argentina. Coordinadora del Programa de Pedagogía Universitaria, Proyecto Acceso e Inclusión Universitaria, y directora del Proyecto UNES 2015-2016, en la Escuela de Ciencia y Tecnología, UNSAM, Argentina. Profesora del Seminario de Pedagogía y Cultura de la Maestría en Educación, Lenguajes y Medios en la Escuela de Humanidades, UNSAM, Argentina.
} 
Resumen: El objetivo de este artículo es analizar las políticas que instrumentan cuatro universidades argentinas, situadas en el conurbano bonaerense, para favorecer el acceso y la permanencia durante el proceso de ingreso y en el primer año de los estudios de grado. Se parte del supuesto de que los factores institucionales -que incluyen la manera en que se organizan los procesos de enseñanza y aprendizaje, el currículo y las estrategias de apoyo a los alumnos- pueden jugar un rol fundamental en la retención estudiantil. El análisis se realiza con base en fuentes secundarias, así como documentos recopilados y entrevistas realizadas a autoridades y coordinadores de los cursos de ingreso de las cuatro instituciones. Los logros y las limitaciones de las políticas -frente al enorme desafío de mejorar en términos de calidad, pertinencia e inclusión- plantean la necesidad de combinar las actuales medidas de apoyo material y pedagógico con un foco más claro en la enseñanza y el currículo, a través de mayores recursos y de un trabajo más estrecho con los docentes en el re-diseño de las estrategias institucionales.

Palabras clave: universidad, acceso a la educación, retención, Argentina

Abstract: The aim of this article is to analyze the policies implemented by four Argentinian universities, located in the Great Buenos Aires area, in order to promote access and permanence during the admission process and in the first year of studies. It is assumed that institutional factors -including how teaching and learning processes, curriculum and student support strategies are organized - can play a key role in student retention. The analysis is based on secondary sources, as well as documents gathered in the four institutions and interviews conducted with authorities and coordinators of the admission courses. The achievements and limitations shown by the policies - with regard to the challenge of improving in terms of quality, pertinence and inclusion — raise the need to combine current measures of material and pedagogical support with a clearer focus on teaching and curriculum issues, through greater resources and closer work with teachers in the re-design of institutional strategies.

Keywords: university, access to education, retention, Argentina

\section{INTRODUCCIÓN}

Este artículo tiene como propósito analizar las políticas que cuatro universidades argentinas implementan para favorecer el acceso y la permanencia, durante el proceso de admisión y en el primer año de estudios ${ }^{4}$. El supuesto que guía el trabajo es que factores institucionales como la organización de los procesos de enseñanza-aprendizaje, el currículo y las estrategias de apoyo a los estudiantes cumplen un rol esencial en la retención estudiantil (Ezcurra, 2011; Juarros, 2006; Pineda-Báez, Pedraza-Ortiz y Moreno, 2011). Como señalan Tinto y Engstrom (2008) para el caso norteamericano, el

4 Este artículo es producto del Proyecto de Investigación Científica y Tecnológica Orientado (PICTO) 2008-0052: "Estrategias institucionales y pedagógicas para aumentar la retención de los alumnos en las universidades del conurbano bonaerense", dirigido por Ana Cambours de Donini, con la financiación de la Agencia Nacional de Promoción Científica y Tecnológica y la Universidad Nacional de San Martín. 
éxito en la incorporación de estudiantes de bajos recursos económicos, en especial cuando no cuentan con una preparación académica adecuada, depende de estrategias proactivas y estructuradas por parte de las instituciones de educación superior.

Teniendo en cuenta las altas tasas de abandono que se registran en el primer año de los programas de educación superior, desde 1980 se consolida -primero en Australia, Estados Unidos, Sudáfrica y varios países de Europa- un abordaje institucional que pone el foco en ese primer año de estudios (Nurt y Calderon citado en Ezcurra, 2011). En el ámbito latinoamericano, la atención a tal problemática es más reciente, pero se han puesto en práctica durante la última década distintas políticas y estrategias para apoyar a los estudiantes en el proceso de incorporación a la universidad y en su tránsito por los primeros tramos de las carreras. Estas medidas abarcan tanto acciones afirmativas hacia poblaciones específicas como otras orientadas a todos los estudiantes, en términos de tutorías académicas, cursos de nivelación y remediales, perfeccionamiento docente, entre otras (Gorostiaga y Cambours de Donini, 2014). Sin embargo, la búsqueda de una mayor inclusión en educación superior, y particularmente en la universidad, plantea una complejidad singular, al tratarse de un nivel con una fuerte tradición de selectividad (Chiroleu, 2009; Juarros, 2006).

En la primera parte del artículo se presentan las tendencias en el plano nacional respecto al ingreso y la retención, lo que incluye el marco de las políticas nacionales recientes y una caracterización general de las políticas institucionales de las universidades públicas. La segunda parte se enfoca en los casos de cuatro universidades situadas en la región del conurbano bonaerense o Gran Buenos Aires: Universidad Nacional de General Sarmiento (UNGS), Universidad Nacional de Lanús (UNLa), Universidad Nacional de San Martín (UNSAM) y Universidad Nacional de Tres de Febrero (UNTREF). Este apartado sistematiza la organización y estructura de los cursos de ingreso previos al inicio de las carreras, así como las políticas de apoyo a la permanencia durante el período de ingreso y el primer año de estudios. ${ }^{5}$ Por último, en el apartado de conclusiones se ofrece una síntesis de los hallazgos y se los relaciona con la discusión sobre la construcción de una universidad más inclusiva.

\section{LAS TENDENCIAS RECIENTES EN ARGENTINA RESPECTO AL INGRESO Y LA RETENCIÓN EN LA UNIVERSIDAD}

\section{Las políticas nacionales}

El sistema de educación superior argentino comprende un sector universitario y un sector de institutos terciarios. El sector universitario, a su vez, abarca universidades e institutos universitarios de gestión pública y de gestión privada. En este trabajo, el interés está

5 No se incluyen en el análisis las acciones de vinculación con el nivel secundario, ni las de becas y bienestar estudiantil. 
centrado en el subsistema de universidades públicas financiadas por el Estado nacional — denominadas universidades nacionales-, que incluye un total de 53 instituciones.

En Argentina, en el año 2011 —último año con cifras oficiales disponibles-, la tasa bruta de educación superior, tomando la población de entre 18 y 24 años, alcanzó $52,6 \%$, mientras que la tasa para el sector universitario llegó a casi 38 \% (Secretaría de Políticas Universitarias, 2011). Si bien en los últimos años la tasa de crecimiento del sector privado ha sido mucho más alta que la del público, en 2011 un $77 \%$ de la matrícula universitaria de pregrado y grado correspondía a las universidades nacionales (calculado a partir de datos de Secretaría de Políticas Universitarias, 2011).

Las tasas netas de escolarización universitaria según ingresos per cápita señalan que el porcentaje de alumnos provenientes de familias con ingresos altos doblan a aquellos que provienen de familias con ingresos bajos (García de Fanelli y Jacinto, 2010, p. 68) ${ }^{6}$. Según datos recientes, la tasa de deserción en la educación universitaria se sitúa cerca del 60 \% (Marquina, 2011). Hay pocos estudios en el país respecto a los factores asociados tanto al acceso como a la permanencia en la universidad. Sin embargo, los datos estadísticos disponibles señalan que los más altos índices de abandono se registran durante el primer año de la carrera, son menores en segundo año y mucho más bajos en los años siguientes. Asimismo, los niveles de deserción son más significativos para los alumnos de bajo estatus socioeconómico (Gessaghi y Llinás, 2005).

Desde fines de la década de 1940 — con excepción de los períodos dictatoriales, en los que se tendió a restringir el ingreso- las políticas públicas han promovido el acceso a la universidad a través de la creación de instituciones, de la gratuidad y del ingreso directo (con el único requisito del título de nivel secundario) dentro del sector estatal. La Ley de Educación Superior (LES), sancionada en 1995, determinó la libertad de las universidades nacionales $-y$ de las unidades académicas en el caso de instituciones con más de 50.000 alumnos - para establecer sus propias políticas y procedimientos de admisión, lo que generó la posibilidad de sistemas no irrestrictos. Al mismo tiempo, abrió la posibilidad del arancelamiento de los estudios de grado, siempre que los recursos que se obtuvieran por esta vía se utilizasen para el otorgamiento de becas. Sin embargo, "la fuerte oposición a esta medida entre los alumnos, las autoridades universitarias y los partidos políticos, tornó inoperante" dicho arancelamiento (García de Fanelli, 2011, p. 40).

Durante la década de 1990 se establecieron diez nuevas universidades nacionales -entre ellas, las cuatro que son parte de este estudio- que se sumaron a las 28 preexistentes. Desde 2003 hasta 2014 se crearon otras quince, mayormente en lugares donde no existía oferta. De ese total de 25 nuevas casas de estudio, doce se localizan en el conurbano bonaerense ${ }^{7}$. Algunos de los motivos que fundamentaron estas

6 No obstante, García de Fanelli (2011) muestra que la brecha en el acceso a la educación superior entre el estrato socioeconómico más alto y el más bajo se redujo levemente en el período 2005-2010.

7 La región metropolitana de Buenos Aires (RMBA) está compuesta por la Ciudad Autónoma de Buenos Aires (CABA) y 24 partidos del conurbano bonaerense o Gran Buenos Aires (GBA). Su población ronda los 14 millones de habitantes, 
creaciones fueron la descentralización de las macrouniversidades (en particular la Universidad de Buenos Aires) y la conveniencia de implementar estructuras más ágiles, una oferta curricular innovadora que atendiera áreas vacantes y una mayor articulación con las necesidades locales. Si bien es cierto que en muchos casos esta política no se enmarcó en una planificación cuidadosa del sistema (Chiroleu, 2012), y que puede haber cuestionamientos sobre la calidad de algunas instituciones (García de Fanelli, 2014), no hay dudas de que ha generado un aumento significativo en las posibilidades de acceso a la universidad.

Por otra parte, durante las dos últimas décadas, ha comenzado a aparecer en la agenda de las políticas nacionales de educación superior la preocupación por los altos índices de abandono. A la adjudicación de becas se la ha acompañado con acciones que buscan articular mejor la escuela secundaria con la educación superior, así como con otras medidas que se proponen facilitar la permanencia de estudiantes de sectores desfavorecidos (Arias et al., 2012). Así, por ejemplo, desde 2005 los Proyectos de Mejoramiento de la Secretaría de Políticas Universitarias (SPU), dirigidos en especial a las carreras de grado que son parte de los procesos de acreditación por parte de la Comisión Nacional de Evaluación y Acreditación Universitaria (CONEAU), han incluido sistemas de tutorías dirigidos a alumnos ingresantes y una mejora de la formación pedagógica de los docentes de primer año. En la misma línea, en el año 2010 se convocó por primera vez a las universidades nacionales a presentar programas de apoyo para implementar acciones complementarias a las Becas Bicentenario - destinadas a carreras científicas y técnicas_- a fin de mejorar el rendimiento de los alumnos de primer año y promover la plena incorporación de los estudiantes a la vida universitaria.

\section{Las políticas institucionales}

Dentro del conjunto de las universidades nacionales, existe una variedad de mecanismos y políticas referidas al acceso a la universidad, asentada en la autonomía que establece la LES. Las distintas modalidades de ingreso en las universidades públicas se agrupan en tres categorías (Sigal y Dávila, 2004):

lo que representa el $35 \%$ del total de la población del país (Rofman, 2014). Sin embargo, la CABA y el GBA se distinguen por su estructura socioeconómica: según datos del año 2010, mientras la Ciudad Autónoma de Buenos Aires tenía 68.773 hogares con necesidades básicas insatisfechas (NBI), los 24 partidos del conurbano poseían 270.962 hogares con NBI. Esto significa una población de 198.114 habitantes con NBI en CABA contra 1.219 .700 en el GBA (Instituto Nacional de Estadística y Censos, 2010). 
1) Ingreso irrestricto

1.1 Sin preingreso

1.2 Con cursos de apoyo y nivelación

1.3 Con cursos y exámenes vinculantes con el plan de estudios

2) Con ingreso mediante pruebas de examen, sin cupo

3) Ingreso mediante prueba y cupo

Ramallo y Sigal (2010) estiman que un 30,8 \% del total de los ingresantes lo hace a través de un examen eliminatorio (modalidades 2 y 3 ).

También es posible distinguir entre barreras formales e informales o implícitas al ingreso. Entre las formales, se puede mencionar a los cursos de ingreso eliminatorios, con cupos y sin cupos, según las carreras y las universidades, mientras que otras instancias curriculares o extracurriculares, supuestamente diseñadas para facilitar el ingreso, se convierten en algunos casos en barreras informales. Por ejemplo, la entrada a la Universidad de Buenos Aires - la institución con más alumnos del sistema- es formalmente directa, pero el primer año de estudios, denominado Ciclo Básico Común, constituye un verdadero filtro informal, ya que solo un $50 \%$ de los alumnos lo terminan en un año (Ramallo y Sigal, 2010, p. 14). Al mismo tiempo, puede considerarse que existen barreras implícitas en todas las instituciones universitarias, que están basadas en exigencias de determinado capital cultural, así como de condiciones y capacidades para hacer frente exitosamente a los desafíos de la experiencia universitaria (García Guadilla, 1991; Juarros, 2006).

Los altos índices de abandono en los cursos de ingreso y en los primeros años de las universidades públicas configuran una tendencia que ha sido subrayada por varios trabajos (Ezcurra, 2007; Marquina, 2011; Parrino, 2010). La ley de 1995 significó la puesta en marcha de nuevas reglas de juego y eso contribuyó a una reflexión por parte de las universidades sobre los problemas de la deserción y el rezago estudiantil (Marquina, 2011). Algunas respuestas a estos replanteos, particularmente entre las nuevas universidades del conurbano, fueron la introducción de cursos preuniversitarios, en los que entran en tensión los objetivos de nivelar conocimientos y aptitudes de los aspirantes e introducirlos a la vida universitaria, con los procesos selectivos (Marquina, $\left.2011^{8}\right)$.

Respecto a las acciones para mejorar la retención, estas parecen ir ganando lugar dentro de las universidades nacionales, en muchos casos como respuesta a los

8 El trabajo de Marquina (2011), enfocado en los casos de las universidades nacionales de General Sarmiento, La Matanza, Lanús, Quilmes y San Martín, hace hincapié en el carácter selectivo de sus mecanismos de admisión, y los diferencia de los sistemas tradicionales de ingreso irrestricto. 
programas impulsados por la SPU y con algún financiamiento (aunque sea inicial) de esa dependencia (Capelari citado en García de Fanelli, 2014; Duarte, 2013). Algunos ejemplos de estas acciones se describen sintéticamente a continuación:

- El Proyecto Niveles, que se llevó adelante en algunas de las asignaturas del Área de Matemáticas de la Facultad de Ingeniería de la Universidad del Centro de la Provincia de Buenos Aires, buscaba lograr la inserción gradual de los alumnos al contemplar tiempos diferenciados para los aprendizajes (que los alumnos pudieran avanzar a diferentes ritmos de acuerdo con sus posibilidades, y retomar los niveles en los que tuvieron dificultades en un primer momento). Se partía del diagnóstico de un desfasaje entre las normas, los hábitos de estudio y las exigencias de la enseñanza media y de la universidad. El proyecto tuvo un impacto positivo al mejorar la retención y acortar los tiempos de acreditación de las asignaturas, lo que aumentó la cantidad de alumnos que promocionaron materias (Aisenson et al. citado en Kisilevsky, 2002).

- El Programa de Ingreso y Permanencia de la Facultad de Ciencias Humanas de la Universidad Nacional del Centro de la Provincia de Buenos Aires, implementado desde el año 2000, incluye, por un lado, tutorías y talleres optativos sobre cuestiones relativas al desempeño académico para los estudiantes (tanto ingresantes como avanzados) y, por otro lado, espacios de reflexión sobre sus prácticas para los docentes. Se destaca que las tutorías de estudio, a cargo de docentes auxiliares, han tenido éxito desde la perspectiva de los estudiantes en términos de adquisición de herramientas para la comprensión de textos y de técnicas de estudio (Baldoni et al., 2007).

- El Programa de Mejoramiento de la Calidad Educativa y Retención Estudiantil de la Universidad del Comahue (Martínez, Echenique y Reta, 2006) partía de un diagnóstico de alto nivel de abandono durante el transcurso del primer año. Sus causas eran atribuidas a una variedad de factores causales (internos y externossocioeconómicos) y a falta de conocimientos, competencias cognitivas y estrategias de aprendizaje requeridas para el nivel universitario. Los objetivos del programa eran: promover la retención de alumnos en primer año; generar diagnósticos enfocados a estos alumnos; conformar equipos docentes en cada 
unidad académica; elaborar estrategias institucionales que apuntaran a mejorar la retención y la calidad educativa en primer año. Las acciones durante 2004-2005 incluyeron: seminarios-taller de formación docente sobre el "oficio" del docente; tutorías (a cargo de docentes y de alumnos avanzados) en tres dimensiones: académica, institucional y socio-relacional (Enríquez y Reta, 2006), con variantes de acuerdo con las diferentes unidades académicas (talleres de escritura, clases de consulta, abordaje de textos, técnicas de estudio, asistencia individual); elaboración de materiales sobre el concepto y la práctica del "oficio" de alumno; seguimiento académico de los estudiantes participantes. Entre los problemas encontrados se señalaron: falta de tiempo o de recursos para movilizarse por parte de los estudiantes; dificultades de comunicación fluida y efectiva entre docentes y estudiantes; insuficiencia de espacios físicos para la actividad tutorial (Martínez, Echenique y Reta, 2006).

- El Régimen de Asesorías de la Facultad de Ingeniería de la Universidad Nacional de Mar del Plata (Moro, Pirro y Viau, 2009) comenzó a implementarse en el año 2008, financiado en parte por el Proyecto de Mejoramiento de la Enseñanza de las Ingenierías (PROMEI) de la SPU. Del seguimiento de una muestra de alumnos ingresantes surgió la identificación de dificultades en la organización del tiempo y el uso de técnicas para el estudio, en la solicitud de becas, en la realización de trámites administrativos, en el uso de la biblioteca y en la vinculación con los docentes. Además, fueron relevados algunos conflictos en la implementación de las asesorías o tutorías (asesores que no cumplían con sus funciones, planteamientos de problemas que excedían las capacidades el ámbito de acción de los asesores) y se propusieron acciones en respuesta a varios de los problemas identificados.

- La Universidad Nacional de Córdoba -en el marco del Proyecto de Apoyo para el Mejoramiento de la Enseñanza en Primer Año de Carreras de Grado de Ciencias Exactas y Naturales, Ciencias Económicas e Informática (PACENI) de la Secretaria de Políticas Universitarias - instrumentó, a partir del año 2009, un sistema de tutorías a cargo de estudiantes avanzados en las carreras de cuatro facultades (Duarte, 2013). El proyecto apuntó al "mejoramiento de la inserción y 
la promoción de los estudiantes ingresantes, y la disminución de los valores en los índices de abandono de los estudios universitarios en el primer año de la carrera" (Ambroggio, 2013, p. 281). Se identificaron efectos positivos importantes, como facilitar la integración a la institución y una mejor comprensión de mecanismos académicos y administrativos, así como la "objetivación" de dinámicas institucionales que condujeron a cambios en las prácticas de enseñanza de algunos docentes y en la flexibilización de instancias evaluativas, entre otros. A su vez, se reconocieron limitaciones de la experiencia, en particular la separación, como circuitos paralelos, de la función tutorial respecto al dictado de las materias por parte de los profesores, que generó tensiones o falta de articulación (Ambroggio, 2013).

\section{LAS POLÍTICAS Y ESTRATEGIAS EN CUATRO UNIVERSIDADES DEL CONURBANO BONAERENSE}

Las cuatro universidades estudiadas se caracterizan por ser de un tamaño relativamente pequeño dentro del conjunto de universidades nacionales argentinas —para el año 2011, la cantidad de alumnos variaba entre 5978 (UNGS) y 12587 (UNSAM) —, pero con tasas de crecimiento anual significativamente más altas que el promedio del sistema (Secretaría de Políticas Universitarias, 2011). Si bien no hay estadísticas fehacientes sobre el nivel de abandono estudiantil en estas cuatro instituciones, la alta proporción de estudiantes que se matriculan sin haber aprobado ninguna materia durante el año anterior (tabla $n . \stackrel{0}{1}$ ) puede ser tomada como indicador de una fuerte presencia de trayectorias irregulares que conllevan el riesgo de la deserción.

Tabla N. 01

Reinscriptos de carreras de pregrado y grado que no han aprobado ninguna materia el año anterior, según institución. Año 2011

\begin{tabular}{lccc}
\hline Universidad & Reinscriptos & $\begin{array}{c}\text { 0 Materias } \\
\text { (frecuencia) }\end{array}$ & $\begin{array}{c}\text { 0 Materias } \\
\text { (porcentaje) }\end{array}$ \\
\hline General & 4720 & 1602 & 34 \\
Sarmiento & & & \\
Lanús & 8882 & 3708 & 42 \\
San Martín & 8926 & 2336 & 26 \\
Tres de Febrero & 5925 & 832 & 14 \\
\hline
\end{tabular}

Fuente: Elaboración propia de los autores

basada en Secretaría de Políticas Universitarias (2011, p. 98, cuadro 2.1.7.a) 
A continuación, se describe la estructura y organización del ingreso en las cuatro universidades, así como las principales acciones de apoyo a la retención en los cursos de ingreso y en el primer año de las carreras. Para ello se utilizan algunas fuentes secundarias, así como documentación recopilada en las instituciones y entrevistas realizadas a integrantes de las secretarías académicas y responsables de los cursos de ingreso en los años 2011 y 2012.

\section{Universidad Nacional de General Sarmiento (UNGS)}

El mecanismo de ingreso a todas las carreras (estudios de pregrado: tecnicatura o mención específica; estudios de grado: licenciaturas, profesorados universitarios, ingenierías) es el Curso de Aprestamiento Universitario (CAU). Su propuesta está orientada a que los estudiantes: a) refuercen sus habilidades básicas de lectura y escritura; b) refuercen sus habilidades de aprendizaje de herramientas matemáticas y de razonamiento matemático; c) se aproximen a la comprensión de la naturaleza de la actividad y el conocimiento científico, y desarrollen nociones y algunas prácticas elementales propias de la producción de dicho conocimiento; y d) adquieran un desenvolvimiento acorde a los requerimientos de la vida universitaria.

EI CAU está conformado por tres asignaturas comunes a todas las carreras: Taller de Lectoescritura, Taller de Matemática y Taller de Ciencia. Las clases (menos para los alumnos que rinden libre) tienen carácter obligatorio, con el requisito de un $75 \%$ de asistencia y aprobación de al menos una materia con promedio de 7 o más, y las otras dos materias con promedio de 4 o más. Las asignaturas son independientes entre sí y pueden cursarse en forma separada por semestres. La condición de aprobación de cada una de ellas es perdurable. Tampoco se pierde la regularidad de ninguna asignatura por el período que comprende los tres turnos consecutivos de exámenes finales luego de la regularización. En estos períodos, se puede recursar solo la asignatura pendiente.

EI CAU tiene tres modalidades de aprobación. La modalidad de cursada cuatrimestral se dicta de marzo a junio en el primer cuatrimestre y de agosto a noviembre en el segundo. La modalidad de cursada intensiva se dicta durante el verano y está dirigida a los estudiantes que adeudan alguna materia de la cursada cuatrimestral o que cumplen con determinados requisitos (por ejemplo, haber aprobado el ingreso a otra universidad o haber aprobado tres materias de una carrera universitaria). En la modalidad libre, deben aprobarse por examen las asignaturas Taller de Lectoescritura y Matemática, y cursarse obligatoriamente el Taller de Ciencia en alguno de los momentos en que se dicta durante el año lectivo.

Algunas asignaturas ofrecen a sus estudiantes clases especiales, que se denominan espacios complementarios, en las que se refuerzan o desarrollan actividades particularizadas que les sirven para mejorar su rendimiento. Esas clases se ofrecen en distintos turnos, preferentemente a la mañana y a la tarde. La organización de los 
espacios complementarios depende de cada asignatura y se realiza sobre la marcha, de acuerdo con las necesidades de los estudiantes.

Feeney, Marquina y Rinesi (2011) reportan que cada año ingresan unos 3000 estudiantes al CAU. Las altas tasas de desgranamiento y de deserción han llevado a replantear algunos aspectos en el desarrollo de este curso: se pasó de una modalidad cuasi anual a otra cuatrimestral de mayor intensidad horaria, junto con modalidades alternativas que lo hacen "más flexible y menos expulsivo" (p. 209). A partir de 2011, el CAU ha iniciado una nueva propuesta que incluye cambios en las estrategias pedagógicas. Uno de ellos es la organización del trabajo en equipo de los docentes de las tres asignaturas (Matemática, Lectoescritura y Ciencia) para la producción de materiales, y la posibilidad de compartir trabajos de reflexión e investigación sobre distintas problemáticas en torno al CAU. Las jornadas sobre cuestiones de ingreso, desarrolladas por primera vez en ese año, dan cuenta de algunas problemáticas pedagógicas: "Explorando los procedimientos lógicos en estudiantes preuniversitarios de Matemática", "Errores presentes en las respuestas escritas dadas por estudiantes de Matemática del CAU", "Competencias lectoras literarias en el aprendizaje de las ciencias", "En torno al paratexto. Propuestas de trabajo con dos géneros".

Frente al problema de la deserción en los primeros años de estudio, se implementó un sistema de apoyo material para los alumnos (becas y entrega gratuita de material bibliográfico) y dispositivos de información, orientación y apoyo, a través de "tutorías individuales y grupales, sistemas de asesoramiento a cargo de los investigadores y docentes, talleres sobre técnicas de estudio, amplios horarios de consulta y servicios de orientación general, vocacional y pedagógica" (Feeney et al., 2011, p. 210). Además, en 2009 la UNGS inició una revisión curricular que buscaba, entre otras cosas, evaluar la incidencia que tenía la estructura de los planes de estudio en el abandono estudiantil.

\section{Universidad Nacional de Lanús (UNLa)}

En la UNLa se destacan dos tipos de ingreso que se imparten todos los años. El primero es un curso cuatrimestral que se desarrolla desde fines de agosto a principios de noviembre, con una frecuencia de tres veces a la semana. El segundo tipo de ingreso se denomina intensivo y se dicta desde principios de febrero a mediados de marzo, con una frecuencia de cuatro veces por semana. La asistencia a los cursos es obligatoria.

El curso de ingreso es el mismo para todas las carreras y consta de tres materias: La Universidad en la Argentina; Métodos y Técnicas para los Estudios Universitarios; Materia Introductoria a la carrera respectiva.

La coordinación está centralizada en una Dirección de Pedagogía Universitaria que depende institucionalmente de la Secretaría Académica. Tanto el diseño de los exámenes como los criterios de evaluación son comunes a todas las comisiones. Respecto a las evaluaciones finales, la asignatura de La Universidad en la Argentina y 
la materia introductoria a la carrera tienen exámenes finales, escritos y presenciales. En tanto, la materia Métodos y Técnicas para los Estudios Universitarios tiene como requisito de aprobación la presentación de una carpeta de trabajos prácticos. De acuerdo con las entrevistas, la matrícula se ha visto incrementada en los últimos años. Cabe señalar que para ciertas carreras (Turismo, Diseño y Comunicación Visual, Sistemas) se han establecido cupos de ingresantes, debido a falta de infraestructura o de docentes.

Con respecto a la organización del curso de ingreso, los profesores son convocados por la Dirección de Pedagogía Universitaria. Tal como surge de las entrevistas realizadas, la designación es resistida por algunos profesores, a veces porque no desean ponerse al frente de ingresantes, dada su especificidad, y además porque las horas de ingreso se cobran como las de la carrera. Sin embargo, se ha estipulado un reconocimiento económico para aquellos profesores que realicen y aprueben alguno de los módulos de capacitación docente. Estos módulos son una forma interesante de abordar cuestiones referidas a cómo enfrentar y resolver los problemas comunes a los alumnos del ingreso. Son totalmente virtuales y, entre ellos, se pueden distinguir los siguientes: Docencia Universitaria y Currículo, Práctica docente, Lectura y Escritura Académica, Las TIC y la Enseñanza Universitaria.

Si bien no se cuenta con estadísticas fehacientes, los entrevistados consideran que la deserción es alta en el curso de ingreso. Estiman que, aproximadamente, un $30 \%$ de los inscriptos no lo comienza y un $15 \%$ deserta durante la cursada. Entre las posibles causas, citan las dificultades en la articulación de horarios con las ocupaciones laborales, y, en áreas de aprendizaje, dificultades respecto a la lectura y escritura académicas. Los entrevistados también se refieren a "actitudes expulsivas" de algunos profesores del ingreso.

La universidad ha implementado para el primer año de las carreras un sistema de docentes orientadores, figura creada desde la Secretaría Académica y bajo la coordinación de la Dirección de Pedagogía Universitaria. Constituye un equipo de docentes encargados de fortalecer y sostener los vínculos del ingresante con la institución, de modo de contribuir a una disminución de los niveles de desgranamiento en el primer año. Se dedican a acompañar, informar y motivar a los ingresantes. Se designa un docente orientador por carrera con una dedicación simple; en general, se trata de un graduado reciente de la propia carrera.

\section{Universidad Nacional de Tres de Febrero (UNTREF)}

La organización del curso de ingreso de la UNTREF posee una estructura dentro de la Secretaría Académica de la Universidad, conformada por el secretario académico, la coordinadora general y la asesora pedagógica del curso de ingreso. Además, cuenta con cerca de cien profesores a cargo de los cursos. El curso es general para todas las carreras y se desarrolla a lo largo de cuatro meses, de febrero a julio de cada año. Se dictan las siguientes materias: Metodología de Estudio, Comunicación Oral y Escrita, y 
una tercera materia que puede ser Matemática, Biología, Fsica-Química, entre otras, dependiendo de la carrera que se va a cursar.

La UNTREF cuenta no solo con material de orientación para el alumno, sino también con material de estudio ad hoc para cada materia. La estructura del ingreso cuenta con tutores que se reúnen con los alumnos cada quince días para guiarlos en cuestiones de estudio y de agenda. También se dictan clases de apoyo de cada materia en horarios diferentes a la cursada.

De acuerdo con los entrevistados, a lo largo de los años el ingreso fue "tomando cuerpo" e "institucionalizándose" dentro de la universidad. La institucionalización permitió el cambio de una posición de soledad y separación del resto de la universidad a una situación de participación y articulación. Dio lugar también a una reflexión teórica sobre el ingreso, particularmente en términos institucionales y pedagógicos. A su vez, se fueron implementando distintas modalidades, según las necesidades que se consideraban más urgentes.

Uno de los cambios producidos es que se dispuso la apertura de un Taller de Estudios Universitarios, que se desarrolla durante la primera semana, antes de comenzar con las materias propiamente dichas del curso de ingreso. En ese taller se ofrecen seis clases, de dos horas cada una, repartidas de la siguiente forma: dos clases de Introducción a los Estudios Universitarios, con foco en qué es la universidad; una clase de presentación de las carreras; y tres clases destinadas a la presentación o introducción a cada una de las materias del curso de ingreso. El taller plantea como objetivo central trabajar con los aspirantes acerca del significado de ingresar a estudios superiores universitarios y, en ese marco, avanzar sobre propósitos más específicos, tales como analizar y reflexionar en forma grupal acerca de las derivaciones y consecuencias que tiene para la vida de cada uno dicho ingreso, así como también comenzar a establecer vínculos firmes con la institución (Mundt, Curti y Tommasi, 2011). También se instrumentaron mecanismos más flexibles de evaluación.

Las autoridades reconocen que el problema de la deserción es muy grave. De los 3000 a 3500 alumnos que se anotan al curso de ingreso, solo un $30 \%$ lo completa y comienza el cursado de carreras. Las percepciones de los entrevistados indican que tal desgranamiento se debe a problemas laborales (especialmente los horarios de clase que se superponen al del trabajo) y familiares, así como al nivel de exigencia de los cursos. Todos los entrevistados ponen el acento en que el ingreso no es a una carrera, sino a la universidad. En ese sentido, se desarrollan acciones y contenidos que tengan el objetivo de nivelar, pero, fundamentalmente, de adaptar e incorporar al estudiante a la vida universitaria. Todos quienes interactúan con los aspirantes tienen que tener claro este propósito específico.

Al igual que en la Universidad Nacional de Lanús, los entrevistados consideran que uno de los mayores problemas es que hay docentes que no se comprometen debidamente con el ingreso. En general, reportan que los docentes son poco autorreflexivos y se quejan mucho de la preparación de los aspirantes. En respuesta a 
eso, se están desarrollando algunas acciones concretas para aumentar la comprensión de la problemática y el compromiso por parte de los docentes, a través de charlas sobre los propósitos de los cursos, así como de contratos laborales más largos, entre otras medidas.

\section{Universidad Nacional de San Martín (UNSAM)}

La UNSAM es la única universidad en la que los procedimientos de admisión se efectúan de manera descentralizada. Los cursos de ingreso varían de acuerdo con la unidad académica, en términos de duración y de contenido, aunque todos utilizan la denominación de Curso de Preparación Universitaria (CPU). Existe una materia de carácter obligatorio para todas las unidades académicas, Introducción a los Estudios Universitarios (IEU). La duración del curso varía entre seis semanas y un cuatrimestre.

Por ejemplo, la Escuela de Ciencia y Tecnología (ECyT) tiene dos llamados a ingreso para licenciaturas e ingenierías, en los meses de febrero y julio. Los cursos constan de cinco semanas de duración y comprenden tres materias: Matemática Básica, IEU y Seminario de Lectoescritura. Para las tecnicaturas universitarias (cuatro en total) se ofrece un ingreso cuatrimestral que se abre en los meses de marzo y julio/agosto (según vacaciones de invierno), que consta de cinco materias: Matemática, IEU, Física, Química y Biología o Informática, según la tecnicatura elegida.

Los objetivos del CPU de la ECyT son: introducir al estudiante en la vida universitaria; interiorizarlo sobre los fines y objetivos específicos de la UNSAM; orientarlo en las áreas decisivas de cada carrera; familiarizarlo con el método de los estudios universitarios; nivelar sus conocimientos para adecuarlos a los requerimientos universitarios; facilitar su transición entre los niveles secundario y universitario, de modo de contribuir a su permanencia disminuyendo dudas vocacionales o situaciones de desaliento a las que puede enfrentarse en la primera etapa de las carreras.

La ECyT ofrece una opción más a los aspirantes: el examen de admisión. Dicho examen consta de dos partes: 1) Matemática y 2) Introducción a los Estudios Universitarios y Seminario de Lectoescritura. Antes de las pruebas, se ofrecen espacios de consulta a cargo de profesores del CPU en los que los aspirantes pueden plantear dudas y ejercitarse con los materiales que se ofrecen ad hoc. Los alumnos aspirantes tienen la oportunidad de dar los exámenes, que se aprueban con una nota mínima de 6, y ahorrar un cuatrimestre de cursada, ya que una vez aprobados pueden comenzar a cursar materias de primer año de cada carrera. En caso de no aprobar, tienen la opción de cursar el ingreso regular de un cuatrimestre.

Por otra parte, en la Escuela de Economía y Negocios (EEyN) y la Escuela de Humanidades (EHU), el CPU se dicta a partir del mes de febrero y tiene una extensión de entre cinco y ocho semanas, dependiendo de la carrera. En este período se encuentran incluidos clases y exámenes. La frecuencia semanal es de cinco días a la semana en el caso de la EEyN, y de entre tres y cuatro días en el caso de la EHU. 
En la EHU, los objetivos del curso son: introducir al estudiante en la vida universitaria e interiorizarlo sobre los fines y objetivos específicos de la UNSAM; orientarlo en las áreas decisivas de cada carrera; familiarizarlo con el método de los estudios universitarios; diagnosticar el nivel en que se encuentra al iniciar su carrera universitaria; nivelar sus conocimientos para adecuarlos a los requerimientos universitarios. Para el caso de la carrera de Psicopedagogía (EHU), el CPU cumple una función selectiva, ya que ingresan por año cerca de 100 alumnos (la inscripción al CPU en esa carrera suele superar los 200 aspirantes) que se corresponden con los mejores desempeños logrados en las evaluaciones. En la EEyN los objetivos del curso son la ambientación de los estudiantes en la vida universitaria y la nivelación de conocimientos.

Sobre la organización curricular, además de la ya mencionada materia IEU, se dictan entre dos y cuatro materias más, cuyos contenidos y objetivos se corresponden con los específicos de cada carrera. Sobre los docentes a cargo de los cursos, su designación depende de criterios como especificidad de los temas a desarrollar y dedicación horaria del plantel docente, aunque hay algunos casos en los que no se especifica con claridad.

Consultados los entrevistados de ambas escuelas (EHU y EEyN) respecto de las posibles causas de deserción, coinciden significativamente en algunos puntos: dificultades en la adaptación a la vida universitaria, en el nivel de rendimiento de acuerdo con la exigencia de los estudios académicos y problemas para cumplir el horario por exigencias laborales.

El CPU para el Ciclo General en Ciencias Sociales (incluye el primer tramo de licenciaturas de la EHU, de la Escuela de Política y Gobierno, y del Instituto de Altos Estudios Sociales) cuenta con un espacio de tutoría que se implementa desde el año 2008, con el objetivo de ofrecer información, asistencia y acompañamiento permanente a los alumnos en sus primeras experiencias en la universidad. Los tutores -estudiantes avanzados de las carreras - orientan a los alumnos en aspectos metodológicos y organizativos. Se procura que los alumnos mantengan un contacto asiduo con los tutores para disipar todas sus dudas y mantenerse informados sobre las posibilidades que les brinda la universidad. La tutoría cuenta con una página web que contiene información administrativa, información sobre las carreras y recursos para el estudio.

Por otra parte, la EHU organizó durante 2010 —en el marco de un proyecto de mejora financiado por el Proyecto de Apoyo a las Carreras de Ciencias Humanas (PROHUM) de la SPU - tutorías con un propósito similar, orientadas a estudiantes de las carreras de Educación, Filosofía y Psicopedagogía — tanto para el CPU como para el primer año-que fueron discontinuadas por falta de fondos.

La ECyT, en tanto, desarrolla desde 2011 un Programa de Mejora de la Enseñanza (PME), con foco en los profesores del CPU y de las asignaturas de primer año. El PME tiene como objetivos: mejorar las condiciones de enseñanza en el período de acceso de los aspirantes a las carreras de la escuela; mejorar la enseñanza para optimizar la regularidad y continuidad de las trayectorias de los estudiantes (acceso, 
retención, progreso); promover la formación de equipos docentes y construcción de estrategias de participación; y producir mejoras pedagógicas en el CPU y en carreras de pregrado y grado a partir del diseño de nuevos proyectos de cátedra de los equipos docentes. El programa trabaja sobre proyectos diseñados por distintas cátedras, que ayudan a los docentes a reflexionar sobre los resultados académicos de sus propias materias para que, desde allí, puedan intervenir en una mejora de acuerdo con su propio diagnóstico.

La ECyT también implementa, dentro del área de Pedagogía Universitaria, un Programa de Acceso e Inclusión Universitaria, que aporta un acompañamiento individualizado y orientador, al brindarles a los estudiantes herramientas pedagógicas y académicas para facilitar su inserción en la vida universitaria. Los estudiantes pueden consultar sobre dificultades académicas, orientación general sobre el camino universitario, dudas vocacionales, problemas de horarios, vínculos con compañeros y docentes, perfil de la carrera y el egresado, entre otros. Seguimiento y acompañamiento de los estudiantes, con especial énfasis en los becarios, son sus dos ejes vertebradores.

Como se muestra en la tabla $n .-2$, cada universidad define una estructura curricular para la etapa, curso o ciclo de ingreso. De acuerdo con los componentes curriculares, se constata que en la mayoría de los casos coinciden en centrarse en las habilidades de lectoescritura, producto de reflexiones o diagnósticos previos, según universidad. 
Tabla N.O2

Características de los mecanismos de ingreso en UNGS, UNLa, UNSAM y UNTREF

\begin{tabular}{|c|c|c|c|}
\hline Universidad & Nombre del ciclo & Composición curricular & Modalidad \\
\hline UNGS & $\begin{array}{l}\text { CAU (Curso de } \\
\text { aprestamiento } \\
\text { universitario) }\end{array}$ & $\begin{array}{l}\text { Tres asignaturas: } \\
\text { Taller de Matemática } \\
\text { Taller de Lectoescritura } \\
\text { Taller de Ciencia }\end{array}$ & $\begin{array}{l}\text { Presencial } \\
\text { CAU intensivo de verano } \\
\text { Duración: } 1 \text { cuatrimestre, } \\
\text { salvo modalidad intensiva } \\
\text { Las asignaturas pueden ser } \\
\text { aprobadas a través de un } \\
\text { examen libre }\end{array}$ \\
\hline UNLa & Curso de ingreso & $\begin{array}{l}\text { Tres asignaturas: } \\
\text { La universidad en la Argentina } \\
\text { Métodos y técnicas para los } \\
\text { estudios universitarios } \\
\text { Materia introductoria de la carrera } \\
\text { respectiva }\end{array}$ & $\begin{array}{l}\text { Presencial } \\
\text { Duración: } 1 \text { cuatrimestre o } \\
6 \text { semanas si es en el } \\
\text { verano (febrero-marzo) }\end{array}$ \\
\hline UNTREF & Curso de ingreso & $\begin{array}{l}\text { Taller de estudios universitarios } \\
\text { Tres asignaturas: } \\
\text { Metodología de estudio } \\
\text { Comunicación oral y escrita } \\
\text { Asignatura según carrera }\end{array}$ & $\begin{array}{l}\text { Presencial } \\
\text { Duración: } 1 \text { cuatrimestre }\end{array}$ \\
\hline UNSAM & $\begin{array}{l}\text { CPU (Curso de } \\
\text { preparación } \\
\text { universitaria) }\end{array}$ & $\begin{array}{l}\text { Según Unidad Académica } \\
\text { (Todas coinciden en la asignatura } \\
\text { IEU: Introducción a los estudios } \\
\text { universitarios) }\end{array}$ & $\begin{array}{l}\text { Presencial } \\
\text { Duración variable según } \\
\text { unidad académica } \\
\text { (entre } 5 \text { semanas y un } \\
\text { cuatrimestre) } \\
\text { Opción de examen de } \\
\text { admisión en la ECyT }\end{array}$ \\
\hline
\end{tabular}

Fuente: Elaboración propia de los autores (2016)

El elemento de mayor heterogeneidad en la estructura de los cursos de ingreso es su duración, ya que varían entre un cuatrimestre y cinco semanas, lo que supone una experiencia formativa muy diferente. Por otra parte, además de las asignaturas que se ofrecen en las "cajas" curriculares, las universidades brindan -en mayor o menor medida, según cada institución, y a veces en forma discontinua por falta de financiamiento- una batería de herramientas (tutorías, clases de apoyo, de consulta, orientación, becas, entre otras) que son en algunos casos comunes a todos los estudiantes y que, en otros casos, se focalizan en aquellos considerados en riesgo de interrumpir su trayectoria, abandonar o tener bajo rendimiento, para que, a través de las diferentes estrategias, logren superar los problemas que se les presentan (tabla $n . .03$ ). 
Tabla N.O3

Políticas y estrategias adoptadas por las cuatro universidades para el ingreso y el primer año de estudios

\begin{tabular}{|c|c|c|c|}
\hline Universidad & Coordinación de acciones & Ingreso & Estrategias de apoyo \\
\hline UNLa & $\begin{array}{l}\text { Centralizada. A cargo de la } \\
\text { Dirección de Pedagogía } \\
\text { Universitaria dependiente de } \\
\text { la Secretaría Académica. }\end{array}$ & $\begin{array}{l}\text { Dos tipos de ingreso: curso } \\
\text { cuatrimestral y curso } \\
\text { intensivo para todas las } \\
\text { carreras de la universidad. }\end{array}$ & $\begin{array}{l}\text { Sistema de docentes } \\
\text { orientadores en el primer año } \\
\text { de las carreras. }\end{array}$ \\
\hline UNTREF & $\begin{array}{l}\text { Centralizada. Estructura en la } \\
\text { Secretaría Académica } \\
\text { conformada por secretario } \\
\text { académico, coordinadora } \\
\text { general y asesora pedagógica } \\
\text { de ingreso. }\end{array}$ & $\begin{array}{l}\text { Único curso de ingreso para } \\
\text { todas las carreras. Es } \\
\text { cuatrimestral. }\end{array}$ & $\begin{array}{l}\text { Tutorías y clases de apoyo. } \\
\text { Coordinadores por materia. } \\
\text { Taller de estudios } \\
\text { universitarios. }\end{array}$ \\
\hline UNGS & $\begin{array}{l}\text { Centralizada. Equipo con } \\
\text { dependencia del Instituto de } \\
\text { Desarrollo Humano. }\end{array}$ & $\begin{array}{l}\text { Único curso de ingreso a } \\
\text { todas las carreras: CAU } \\
\text { (Curso de Aprestamiento } \\
\text { Universitario). Tres } \\
\text { modalidades: cuatrimestral, } \\
\text { intensiva y libre. }\end{array}$ & $\begin{array}{l}\text { Becas y distribución gratuita } \\
\text { de material. } \\
\text { Espacios complementarios. } \\
\text { Tutorías. }\end{array}$ \\
\hline UNSAM & $\begin{array}{l}\text { Descentralizada. Cada unidad } \\
\text { académica diseña sus propios } \\
\text { proyectos o programas. }\end{array}$ & $\begin{array}{l}\text { Cada unidad académica } \\
\text { tiene su CPU (Curso de } \\
\text { Preparación Universitaria) } \\
\text { con duraciones variables } \\
\text { entre cinco semanas y un } \\
\text { cuatrimestre, o examen de } \\
\text { ingreso en una unidad } \\
\text { académica. }\end{array}$ & $\begin{array}{l}\text { Programas o proyectos de } \\
\text { cada escuela: tutorías en } \\
\text { Ciclo de Ciencias Sociales. } \\
\text { En Escuela de Ciencia y } \\
\text { Tecnología: Programa de } \\
\text { Mejora de la Enseñanza y } \\
\text { Programa de Acceso e } \\
\text { Inclusión Universitaria. }\end{array}$ \\
\hline
\end{tabular}

Fuente: Elaboración propia de los autores (2016) 


\section{CONCLUSIONES}

Si bien existe una fuerte tradición de ingreso irrestricto en el sistema universitario estatal argentino, durante las últimas dos décadas las políticas institucionales de las universidades nacionales han sido muy heterogéneas en lo que hace a los mecanismos de admisión. En tanto, han comenzado a implementarse experiencias orientadas a favorecer la adaptación a la cultura universitaria de los ingresantes y a brindar apoyos pedagógicos para mejorar la retención, en particular en el primer año de estudios y, en algunos casos, con apoyo de programas de la SPU.

Las universidades del conurbano son parte de un movimiento de ampliación de oportunidades educativas que enfrenta el desafío de mejorar el acceso y la retención de los estudiantes en contextos y con poblaciones distintas a las que son propias, en su mayoría, de las universidades nacionales. Situadas en un entorno social particularmente desfavorable, se encuentran ante los dilemas planteados por la necesidad de modificar formatos académicos y dinámicas de selección implícitas, para dar respuesta a tres exigencias: proveer condiciones para la inclusión de una población estudiantil más heterogénea que la tradicional y con predominio de sectores sociales relativamente desaventajados (tanto en términos de recursos económicos como en su preparación académica); mejorar la calidad de los procesos académicos, que están ligados a la preparación de los alumnos para hacer frente a las obligaciones académicas que cada carrera supone; formar profesionales comprometidos con la sociedad y sus demandas actuales.

El relevamiento realizado en las cuatro universidades estudiadas indica que estas han llevado a cabo intentos por solucionar parte de esos dilemas a partir de la institucionalización de cursos de ingreso, la instrumentación de acciones de apoyo a los alumnos de los primeros años y, en algunos casos, la revisión de los marcos curriculares y las estrategias de enseñanza. En varios casos, los testimonios de las autoridades académicas dan cuenta de un proceso de aprendizaje que ha permitido replantear estrategias, formatos y herramientas con resultados positivos. Por otra parte, es escasa la presencia de programas de la SPU que hayan contribuido con estos procesos.

Se destaca que los cursos de ingreso se proponen, principalmente, preparar a los ingresantes a la vida universitaria y reforzar habilidades y conocimientos necesarios para el inicio de los estudios universitarios, lo cual se orienta a un horizonte de democratización del acceso (Juarros, 2006; Gluz, 2011). El modo de ingreso en las universidades analizadas correspondería a la clasificación de "selectivo con y sin cupo" (Marquina, 2011). Sin embargo, y más allá de que no se reconoce de forma explícita un objetivo de selección en ninguno de los cursos, en la práctica existe un conjunto de apoyos y de posibilidades de recuperación y recursado que relativizan mucho la selectividad, con la excepción de unas pocas carreras que presentan cupos de ingreso.

Los responsables de los cursos de ingreso parten del reconocimiento de un conjunto de condiciones que conspiran contra la permanencia de los ingresantes, que 
abarcan cuestiones externas (en particular, la formación previa y la situación laboral) y aspectos internos como el proceso de socialización, muchas veces difícil, que los estudiantes deben atravesar para incorporarse a pleno a la vida universitaria. El formato de ingreso - con énfasis en aspectos como las habilidades de lectoescritura y mecanismos de orientación institucional y pedagógica - buscaría, entonces, dar respuesta a condicionantes que implican barreras implícitas (García Guadilla, 1991) para el acceso a la universidad. Sin embargo, el corto tiempo del curso en los casos en los que se organiza en solo cinco semanas puede atentar contra la efectividad de esa respuesta.

Por otra parte, dado el rol fundamental que tienen los docentes en el desempeño estudiantil (Ezcurra, 2007; Pineda-Báez et al., 2001), se destaca que las instituciones también encuentran una limitación importante en las "actitudes expulsivas" o la "falta de compromiso" de algunos profesores a cargo del dictado de materias, tanto en el ingreso como en el primer año.

Respecto a las políticas que favorecen la permanencia, medidas como las clases de apoyo o las tutorías por parte de docentes orientadores o estudiantes avanzados han tratado de remediar los altos niveles de abandono en ambas instancias (ingreso y primer año). Sin embargo, a pesar de algunos ejemplos de acciones orientadas a revisar el currículo y las prácticas docentes, las estrategias institucionales que prevalecen suelen estar orientadas más a los alumnos que a los profesores, más a lo extracurricular que a la enseñanza y aprendizaje en el aula, y más a lo que debe agregarse por fuera de la oferta curricular que a la experiencia pedagógica cotidiana, algo que Ezcurra (2011) define como "intervenciones periféricas" que pueden resultar poco eficaces para superar problemas que se dan en las prácticas cotidianas (véase también Gluz, 2011).

Otra parte de la investigación realizada por nuestro equipo (Arias, Mihal, Lastra y Gorostiaga, 2015) también ha mostrado que, mientras que existen percepciones mayormente positivas por parte de los estudiantes respecto a los mecanismos de ingreso y las acciones de apoyo para la permanencia, se cuestionan en algunos casos la efectividad de los cursos de ingreso - en virtud de aspectos como los contenidos y la duración- en preparar a los estudiantes para hacer frente a los desafíos académicos que suponen las primeras materias de las carreras. Por otro lado, existen visiones contrapuestas entre los docentes sobre la necesidad de establecer parámetros más centrados en las realidades socioeducativas y trayectorias de vida de los estudiantes de estas universidades o mantener modelos institucionales y pedagógicos más tradicionales que preservarían la "calidad educativa" (Arias et al., 2015).

Podemos concluir que, con características específicas en cada caso, las cuatro universidades han llevado adelante, desde su creación, un proceso de aprendizaje -no exento de tensiones y contradicciones-en el diseño e implementación de políticas para favorecer el acceso y la permanencia de los estudiantes, particularmente en el primer año de estudios. Los logros y las limitaciones que esas políticas han mostrado, frente al desafío de obtener mejoras en términos de calidad, pertinencia e inclusión, plantean la 
necesidad de combinar las medidas de apoyo material y pedagógico existentes con un foco más claro en la enseñanza y el currículo, a través de un mayor nivel de recursos para lo cual podría ser clave el rol de un actor como la SPU- y de un trabajo más estrecho con los docentes en el re-diseño de las estrategias institucionales.

\section{REFERENCIAS}

Ambroggio, G. (2013). Tutorías: repasar lo aprendido y anotar lo pendiente. En M. E. Duarte (Comp.), Tutorías para ingresantes: experiencias en la UNC (pp. 281-294). Córdoba, Argentina: Universidad Nacional de Córdoba.

Arias, M. F., Bentivenga, J., Coppola, N., Donini, A., Fernández Lamarra, N., Gorostiaga, J.,... Zamboni, P. (2012). La educación superior en Argentina. En Red Iberoamericana de Investigaciones en Políticas Públicas, La educación superior en el MERCOSUR: Argentina, Brasil, Paraguay y Uruguay hoy (pp. 21-113). Buenos Aires, Argentina: Biblos.

Arias, M.F., Mihal, I., Lastra, K., y Gorostiaga, J. (2015). El problema de la equidad en las universidades del Conurbano bonaerense en la Argentina: un análisis de políticas institucionales para favorecer la retención. Revista Mexicana de Investigación Educativa, 20(64), 47-69.

Baldoni, M., Barrón, M.P., Capristo, V., Corrado, R., Eizaguirre, M.D., Fernández, G.,... Laxalt, I. (2007). Las experiencias y las expectativas de aprendizaje de los alumnos universitarios. En Actas de las Segundas Jornadas de Pedagogía Universitaria. San Martín, Argentina: UNSAM Edita.

Chiroleu, A. (2009). La inclusión en la educación superior como política pública: tres experiencias en América Latina. Revista Iberoamericana de Educación, 48(5), 1-15.

Chiroleu, A. (2012). Políticas de educación superior en América Latina en el siglo XXI: ¿inclusión o calidad? Archivos Analíticos de Políticas Educativas, 20(13), 1-20. Recuperado de http://epaa.asu.edu/ojs/article/view/916

Duarte, M.E. (Comp.). (2013). Tutorías para ingresantes: experiencias en la UNC. Córdoba, Argentina: Universidad Nacional de Córdoba.

Enríquez, L., y Reta, L. (2006). La tutoría de pares. Del fast food académico a la construcción de una relación pedagógica singular. Recuperado de http://www.reformadel18.unc.edu.ar/privates/reta.pdf

Ezcurra, A. M. (2007). Los estudiantes de nuevo ingreso. Democratización y responsabilidad de las instituciones universitarias. Cuaderno de Pedagogía Universitaria, 2, 1-57.

Ezcurra, A. M. (2011). Igualdad en educación superior. Un desafío mundial. Los Polvorines, Argentina: IEC-Universidad Nacional de General Sarmiento.

Feeney, S., Marquina, M., y Rinesi, E. (2011). Democratizar la universidad: un ensayo permanente. En N. Fernández Lamarra y M. F. Costa de Paula (Comps.), La democratización de la educación superior en América Latina. Límites y posibilidades (pp. 201-214). Sáenz Peña, Argentina: EDUNTREF. 
García de Fanelli, A. (2011). La educación superior en Argentina 2005-2009. En J.J. Brunner y R. Ferrada Hurtado (Eds.), Educación Superior en Iberoamérica. Informe 2011. Santiago, Chile: CINDAUNIVERSIA.

García de Fanelli, A. (2014). Inclusión social en la educación superior argentina: indicadores y políticas en torno al acceso y la graduación. Páginas de Educación, 7(2), 275-297.

García de Fanelli, A., y Jacinto, C. (2010). Equidad y educación superior en América Latina: el papel de las carreras terciarias y universitarias. Revista Universia, 1(1), 58-75.

Gessaghi, V., y Llinás, P. (2005). Democratizar el acceso a la educación superior. Buenos Aires, Argentina: CIPPEC.

García Guadilla, C. (1991). Modelos de acceso y políticas de ingreso a la educación superior. El caso de América Latina y el Caribe. Educación Superior y Sociedad, 2(2), 72-93.

Gluz, N. (2011). Recapitulación: cuando la admisión es más que un problema de 'ingresos'. En N. Gluz (Comp.), Admisión a la universidad y selectividad social (pp. 231-239). Los Polvorines, Argentina: Universidad Nacional de General Sarmiento.

Instituto Nacional de Estadística y Censos. (2010). Censo de población 2010.

Recuperado de

http://www.indec.gov.ar/nivel4 default.asp?id tema $1=2 \&$ id tema $2=41$ \&id tema $3=135$

Gorostiaga, J., y Cambours de Donini, A. (2014). Políticas y estrategias para la retención en la Educación Superior. En A. Teodoro y J. Beltran (Comps.), Sumando Voces: Ensayos sobre Educación Superior en términos de igualdad e inclusión social (pp. 203-214). Buenos Aires, Argentina: Miño y Dávila.

Juarros, M.F. (2006). ¿Educación Superior como derecho o como privilegio? Las políticas de admisión a la universidad en el contexto de los países de la región. Revista Andamios, 3(5), 69-90.

Kisilevsky, M. (2002). Condiciones sociales y pedagógicas de ingreso a la educación superior en la Argentina. En M. Kisilevsky y C. Veleda, Dos estudios sobre el acceso a la educación superior en la Argentina (pp. 13-84). Buenos Aires, Argentina: IIPE-UNESCO.

Marquina, M. (2011). El ingreso a la universidad a partir de la reforma de los '90: las nuevas universidades del conurbano bonaerense. En N. Gluz (Comp.), Admisión a la universidad y selectividad social (pp. 63-86). Los Polvorines, Argentina: Universidad Nacional de General Sarmiento.

Martínez, S., Echenique, M., y Reta, L. (Comps.). (2006). Programa de Mejoramiento de la Calidad Educativa y Retención Estudiantil (UNCo, 2004-2005). Neuquén, Argentina: Universidad Nacional del Comahue.

Moro, L., Pirro, A., y Viau, J. (mayo, 2009). Implementación de un proyecto de asesorías en la Facultad de Ingeniería de la UNMDP. Presentado en las V Jornadas "La Formación del Profesorado: docentes, narrativas e investigación educativa", Universidad Nacional de Mar del Plata, Mar del Plata, Argentina.

Mundt, C., Curti, C., y Tommasi, C. (2011). Inclusión en los estudios universitarios en el conurbano bonaerense: la construcción de una concepción integral desde una perspectiva de gestión. En N. 
Fernández Lamarra y M. F. Costa de Paula (Comps.), La democratización de la educación superior en América Latina. Límites y posibilidades (pp. 215-231). Sáenz Peña, Argentina: EDUNTREF

Parrino, M. C. (diciembre, 2010). Deserción en el primer año universitario. Desafíos y logros. Ponencia presentada en X Coloquio Internacional sobre Gestión Universitaria en América del Sur, Mar del Plata, Argentina.

Pineda-Báez, C., Pedraza-Ortiz, A., y Moreno, I. (2011). Efectividad de las estrategias de retención universitaria: la función del docente. Educación y Educadores, 14(1), 119-135. Ramallo, M., y Sigal, V. (2010). Los sistemas de admisión de las universidades en la Argentina. Documento de Trabajo no. 255. Buenos Aires, Argentina: Universidad de Belgrano.

Rofman, A. (2014). Territorio, sociedad y política en la Región Metropolitana de Buenos Aires. Los Polvorines, Argentina: Universidad Nacional de General Sarmiento.

Secretaría de Políticas Universitarias. (2011). Anuario de Estadísticas Universitarias. Buenos Aires, Argentina: Secretaría de Políticas Universitarias-Ministerio de Educación de la Nación.

Sigal, V., y Dávila, M. (2004). La cuestión de la admisión a los estudios universitarios en Argentina. En O. Barsky, V. Sigal y M. Dávila (Coords.), Los desafíos de la universidad argentina (pp. 205-222). Buenos Aires, Argentina: Universidad de Belgrano-Siglo XXI.

Tinto, V., y Engstrom, C. (2008). Access without support is not opportunity. Change 40, 46-51. 